110

PECOMA OF THE PELVIC FLOOR: AN UNUSUAL LOCALIZATION

${ }^{1}$ B Alberghetti ${ }^{*},{ }^{2} \mathrm{C}$ Fratus, ${ }^{2} \mathrm{~A}$ Orlandini, ${ }^{3} \mathrm{~A}$ Cominotti, ${ }^{4} \mathrm{E}$ Fogazzi, ${ }^{1} \mathrm{E}$ Sartori, ${ }^{2} \mathrm{D}$ Gatti. ${ }^{1}$ Università degli studi di Brescia, Ginecologia e ostetricia- Spedali Civili di Brescia, Brescia, Italy; ${ }^{2}$ ASST Garda- Ospedale di Manerbio, Ginecologia e ostetricia- Ospedale di Manerbio, Manerbio, Brescia, Italy; ${ }^{3}$ ASST Garda- Ospedale di Manerbio, Anatomia Patologia Ospedale di Manerbio, Manerbio, Brescia, Italy; ${ }^{4}$ ASST Garda- Ospedale di Manerbio, Radiodiagnostica - Ospedale di Manerbio, Manerbio, Brescia, Italy

\subsection{6/ijgc-2021-ESG0.614}

Introduction/Background* Perivascular epithelioid cells tumors (PEComas) are rare mesenchymal neoplasms composed by perivascular epithelioid cells that express both melanocytic and myogenic markers at the anatomopathological and immunohistochemical studies.

PEComas family is composed by angiomyolipomas, pulmonary clear cell "sugar" tumors, lymphangioleiomyomatosis, primary extrapulmonary sugar tumors and clear cell tumors of falciform ligament. In the $25 \%$ of the cases PEComas involve the female genital tract, the uterus is the most likely affected organ and the vagina the least.

This disease is mostly benign, however, there are cases of PEComas with local and distant metastasis at diagnosis. Thus, some risk scores have been developed to predict the benignity, malignancy or uncertain potential of each tumor.

Because of the rarity of such tumors, the diagnosis is usually histological, although recently common sonographic features have been defined.

Treatment is essentially surgical with the possibility of using chemotherapy for locally advanced or recurrent disease.

Methodology

Result(s)* We report a case of a 73 years old woman referred to our hospital for a sense of vaginal weight; the gynecological examination revealed a $4 \mathrm{~cm}$ paravaginal mass. Ultrasound showed a hypoechogenic solid tumor with smooth contour, moderately vascularized (Color Score 3), in the right paravaginal space. The mass was adherent to the vaginal wall and hypomobile to the wall of the rectum and to the pelvic floor with no obvious infiltration of these structures.

The MRI study described a 4-cm-large mass with inhomogeneous vascularization at the level of the right posterolateral vaginal wall that imprinted the right elevator ani muscle without a clear plane of cleavage and, posteriorly, the anterior wall of the rectum with normal plane of cleavage.

The histological examination of the tumor revealed a PEComa. No other localizations were seen in CT scan either in pelvis or in upper abdomen; a total abdominal hysterectomy and bilateral salpingo-ophorectomy was performed to reach the mass located inside the right paravaginal-pararectal space on pubococcigeal muscle. The mass was then totally removed.

Conclusion* We report this case because of the exceptional localization of the PEComa and the peculiarity of the radiological study.

\section{PRIMARY CERVICO-VAGINAL NON-HODKING LYMPHOMA: CASE REPORT AND REVIEW}

M Crespo*, J Amengual, M Ruiz, J Rioja, L Vila, A Torrent. Universitary Son Espases Hospital

\subsection{6/ijgc-2021-ESG0.615}

Introduction/Background* Non-Hodgkin lymphoma (NHL) is an hematologic malignancy and $25 \%$ of NHL patients present with a primary extranodal tumor. Primary female genital tract presentation is extremely rare $(<0,1 \%)$ with only a few cases reported.

\section{Methodology}

Result(s)* 83-year-old, nullipara whom consulted for vaginal bleeding. She used a long-standing pessary as treatment for syntomatic pelvic prolapse. When pessary was removed an exophytic and violet tumor was observed. It involved the proximal vagina and part of cervix.

A transvaginal ultrasound confirmed a well-defined $35 \mathrm{X} 40$ $\mathrm{mm}$ lesion located in proximal vagina. Rectum was not involved.

During the diagnostic process the patient consulted for heavy genital bleeding, uncontrollable with conservative treatment such as packing of the vagina.

Computed tomography was performed. It revealed a solid homogeneous cervical mass of $6 \times 7 \times 6 \mathrm{~cm}$ with a $5 \mathrm{~cm}$ haematoma invading right parametria without observing active bleeding and uterine arteries embolization was performed by the Intervencional Radiology Unit achieving correct hemostasis.

Histopathological diagnosis was obtained after a punch vaginal biopsy and reported clonal plasmablastic large cell neoplasia, suggestive of intensely positive Epstein - Barr virus.

PET/CT showed the known mass in the cervico-uterine region with intense associated hypermetabolism, without visualizing any pelvic adenopathies or in other locations with abnormal metabolism.

Hematologists reviewed the patient and recommended chemotherapy. The patient is currently awaiting the treatment and coordinate follow-up care.

Conclusion* Although the primary cervicovaginal lymphoma has an extremely low prevalence, it should be included in the differential diagnosis of gynecologic malignancies.

Radical surgery does not play a role in the treatment of this malignancy since the mainstays of treatment are cytotoxic chemotherapy, monoclonal antibodies and/or radiation therapy. Fortunately, the vast majority of women are diagnosed as early stage disease and characterized by a very high sensitivity to chemotherapy and favorable prognosis. 